\title{
Über die quantitative Bestimmung der Oxalsäure im Harn.
}

Von

Hugh MacLean, M. D. aus Aberdeen, Carnegie Research Fellow.

(Aus der chemischen Abteilung des pathologischen Instituts der Universität zu Berlin.) (Der Redaktion zugegangen am 2. April 1909.)

Vor einigen Jahren haben Autenrieth und Barth ${ }^{1}$ ) eine Methode zur Bestimmung der Oxalsäure im Harn beschrieben, welche im wesentlichen in folgendem besteht.

Man mischt die Tagesmenge Harn mit Chlorcalcium im Überschuß, dann mit Ammoniak bis zur stark alkalischen Reaktion, läßt 18-20 Stunden stehen, filtriert. Den abfiltrierten und ausgewaschenen Niederschlag löst man in etwa $30 \mathrm{ccm}$ einer etwa 15\% igen Salzsäure. Die erhaltene Lösung schüttelt man 4-5 mal mit 150-200 ccm Äther, der 3\% Alkohol absolutus enthält, aus. Der Äther nimmt die Oxalsäure auf, die beim Verdunsten desselben unter Wasserzusatz zurückbleibt und dann in der üblichen Weise als Calciumoxalat in schwach essigsaurer Lösung ausgefällt wird.

Gegenüber dem von E. Salkowski²) angegebenen Verfahren des direkten Ausschüttelns des eingedampften und mit Salzsäure angesäuerten Harns mit Äther soll nach den genannten Autoren ihre Methode eine Reihe von Vorzügen haben.

1. Man kann leicht eine größere Menge Harn in Arbeit nehmen, während bei dem Verfahren von E. Salkowski, wenn man größere Mengen anwendet, die Gefahr der Bildung von Oxalursäure besteht, durch welche ein Teil der Oxalsäure der Bestimmung entzogen wird.

2. Die Oxalsäure wird aus einer nicht zu verdünnten Lösung ausgeschüttelt, was für den Übergang derselben in den Äther wichtig ist.

3. Man bestimmt nur die präformierte Oxalsäure.

1) Diese Zeitschrift, Bd. XXXV, S. 327.

2) Diese Zeitschrift, Bd. XXXI, S. 437. 
Daß die Möglichkeit, größere Mengen Harn anzuwenden, ein Vorzug ist, insofern man eine größere Quantität oxalsauren Kalk resp. Calciumoxyd zur Wägung bekommt, muß ohne weiteres zugegeben werden, denn der Einwand, daß sich beim Eindampfen großer Mengen Harn Oxalursäure bilden könnte, ist nicht ganz von der Hand zu weisen. E. Salkowski hat selbst auf diese Möglichkeit hingewiesen. Dagegen können die Behauptungen $\mathrm{zu}$ 2. und 3. nicht anerkannt werden, denn auch bei dem Verfahren von E. Salkowski erfolgt die Ausschüttelung aus einem kleinen Volumen, meistens noch kleinerem als bei Autenrieth und Barth, ebenso wird bei demselben auch nur die präformierte 0 xalsäure bestimmt, nicht etwa abgespaltene. Wie leicht ersichtlich, steht und fällt das Verfahren von Autenrieth und Barth mit der Vollständigkeit der Fällung des oxalsauren Kalks direkt aus dem Harn. Ist eine solche vollständige Fällung nun mit Sicherheit zu erwarten oder erwiesen? Der Zweifel daran ist naheliegend. E. Salkowski hat schon früher beobachtet, wie die Verfasser auch angeben, daß unter gewissen Umständen regelmäßig Oxalsäure im Filtrat der Fällung des mit Kalkmilch alkalisierten Harns mit Chlorcalcium vorhanden ist, nämlich wenn man den Harn nur sch wach alkalisiert und kurze Zeit (etwa 15 Minuten) nach der Fällung filtriert. Autenrieth und Barth bezweifeln die Richtigkeit dieser Angaben nicht, haben sich aber überzeugt, daß, wenn man genau nach ihren Angaben verfährt d. h. bei der Fällung stark mit Ammoniak alkalisiert und erst nach 18-20 Stunden filtriert, im Filtrat keine $0 x$ alsäure nachzuweisen ist.

Diesen Punkt habe ich auf Veranlassung von Prof. E. Salkowski und unter seiner Leitung, für die ich meinen besten Dank ausspreche, nachgeprüft und gleichzeitig untersucht, ob man nach dem Verfahren von E. Salkowski in der Tat weniger Oxalsäure erhält, wenn man den Harn sehr stark eindampft. Zur Prüfung des Filtrates der Chlorcalciumfällung wurde dasselbe eingedampft, mit Salzsäure angesäuert und mit

1) Diese Zeitschrift, Bd. X, S. 121 und E. Salkowski, Practicum der physiol. Chemie, 1. Aufl. (1893), S. 208. Später ist festgestellt worden, daß dasselbe auch bei Fällung mit Ammoniak und Chlorcalcium der Fall ist. 
alkoholhaltigem Äther ausgeschüttelt. Das Schütteln wurde jedesmal sehr energisch und anhaltend (mit der Hand) bewirkt und jedesmal 5 Ätherextraktionen gemacht. Es kam nur menschlicher Harn zur Anwendung, mit Chloroform konserviert. Bei der kurzen Zeit, welche die Verarbeitung einer jeden Gruppe von Versuchen nur in Anspruch nahm, hat man eine Änderung des Oxalsäuregehaltes beim Stehen nicht zu befürchten.

Der Versuche ergaben nun folgendes:

\section{Harn I.}

a) $500 \mathrm{ccm}$ wurden auf $150 \mathrm{ccm}$ eingedampft, dann nach E. Salkowski verfahren. Erhalten $8,32 \mathrm{mg} \mathrm{CaC}_{2} \mathrm{O}_{4} \cdot{ }^{1}$ )

b) $500 \mathrm{ccm}$ nach Autenrieth und Barth, 18 Stunden stehen gelassen. Erhalten $6,21 \mathrm{mg} \mathrm{CaC}_{2} \mathrm{O}_{4}$.

Im Filtrat konnte oxalsaurer Kalk nachgewiesen werden. Die Ausscheidung zeigte anfangs nicht die typische Krystallform, wohl aber, als die überstehende Flüssigkeit abgegossen und der Niederschlag nochmals mit Salzsäure, Ammoniak und Essigsäure behandelt wurde. Wie hier gleich bemerkt werden soll, waren auch sonst nicht immer die typischen quadratischen Oktaëder vorhanden, sondern einigemal die von Feser und Friedberger ${ }^{2}$ ) beschriebenen quadratischen Prismen mit pyramidalen Endflächen.

\section{Harn II.}

a) $500 \mathrm{ccm}$ nach dem Verfahren von E. Salkowski behandelt, gaben $7,98 \mathrm{mg} \mathrm{CaC}_{2} \mathrm{O}_{4}$.

Je $500 \mathrm{ccm}$ wurden nach dem Verfahren von Autenrieth und Barth behandelt, jedoch verschieden lange stehen gelassen. In jedem Fall wurde auch die Quantität der Oxalsäure im Filtrat bestimmt. Die Resultate sind in folgender Tabelle zusammengestellt.

Der Fehler bei Nichtberücksichtigung des Filtrates beträgt also rund $1 / 4$ des Gehaltes, resp. 35\% der aus dem Niederschlag erhaltenen Oxalsäure; man könnte sogar daran denken, diese Zahl als Korrektur einzuführen, wenn man kein genaueres Verfahren hätte.

1) Aus $\mathrm{CaO}$ zurückberechnet.

2) Malys Jahresber., Bd. IV, S. 231. 


\begin{tabular}{|c|c|c|c|c|c|}
\hline \multirow{2}{*}{$\begin{array}{l}\text { Versuchs- } \\
\text { nummer }\end{array}$} & \multirow{2}{*}{\begin{tabular}{|} 
Zeit des \\
Stehen- \\
lassens vor \\
dem Filtrieren \\
in Stunden
\end{tabular}} & \multicolumn{3}{|c|}{ Calciumoxalat in $\mathrm{mg}$} & \multirow{2}{*}{$\begin{array}{l}\text { Oxalsäuregehalt } \\
\text { des Filtrates in } \\
\text { Prozenten der } \\
\text { gesamten } \\
\text { Uxalsäure }\end{array}$} \\
\hline & & $\begin{array}{l}\text { aus dem } \\
\text { Nieder- } \\
\text { schlag }\end{array}$ & $\begin{array}{c}\text { aus dem } \\
\text { Filtrat }\end{array}$ & $\begin{array}{c}\text { zu- } \\
\text { sammen }\end{array}$ & \\
\hline 1 & 20 & 6,26 & 2,65 & 8,91 & 29,7 \\
\hline 2 & 48 & 6,43 & 2,01 & 8,44 & 23,8 \\
\hline 3 & 72 & 5,94 & 2,25 & 8,19 & 27,5 \\
\hline 4 & 96 & 6,52 & 2,12 & 8,64 & 24,6 \\
\hline Mittel & - & 6,29 & 2,26 & 8,55 & 26,4 \\
\hline
\end{tabular}

Gleichzeitig ist bemerkenswert, daß dieses Verfahren der doppelten Untersuchung einen etwas höheren Wert gegeben hat als die Bestimmung nach E. Salkowski. Ob dies nur Zufall, mußte weiter geprüft werden.

\section{Harn III.}

A. $500 \mathrm{ccm}$ nach Autenrieth und Barth ausgefällt, dann aber nicht abfiltriert, sondern zur Trockene eingedampft, dann in Salzsäure gelöst und mit Äther ausgeschüttelt.

a) $8,68 \mathrm{mg} \mathrm{CaC}_{2} \mathrm{O}_{4}$.

b) $8,94 \mathrm{mg} \mathrm{CaC}_{2} \mathrm{O}_{4}$.

B. $500 \mathrm{ccm}$ nach Autenrieth und Barth

a) $6,75 \mathrm{mg} \mathrm{CaC}_{8} \mathrm{O}_{4}$ im Niederschlag $+2,35$ aus dem Filtrat $=9,10 \mathrm{mg}$.

b) $6,43 \mathrm{mg} \mathrm{CaC}_{2} \mathrm{O}_{4}$ im Niederschlag $+2,08$ aus dem Filtrat $=8,51 \mathrm{mg}$.

C. Nach E. Salkowski

a) $500 \mathrm{ccm}$ bis auf $60 \mathrm{ccm}$ eingedampft, gaben $9,01 \mathrm{mg} \mathrm{CaC}_{2} \mathrm{O}_{4}$.

b) $500 \mathrm{ccm}$ bis zum Sirup eingedampft, gaben $9,31 \mathrm{mg} \mathrm{CaC}_{2} \mathrm{O}_{4}$ :

Es scheint demnach, daß es wenigstens bei Anwendung von nicht mehr als $500 \mathrm{ccm}$ Harn nicht zur Bildung von Oxalursäure kommt. Die bei dem abgeänderten Fällungsverfahren erhaltenen Werte sind fast dieselben, wie die nach dem Verfahren von E. Salkowski erhaltenen.

Harn IV.

a) $500 \mathrm{ccm}$ nach E. Salkowski gaben $10,40 \mathrm{mg} \mathrm{CaC}_{2} \mathrm{O}_{4}$. 
b) $500 \mathrm{ccm}$ nach Autenrieth und Barth, jedoch mit dem Niederschlag fast bis zur Trockene eingedampft, 9,98 $\mathrm{mg} \mathrm{CaC}_{2} \mathrm{O}_{4}$.

Nach diesen Ergebnissen müssen wir die Richtigkeit der Angabe von Autenrieth und Barth, daß das Filtrat vom Niederschlag keine Oxalsäure enthalte, bestreiten; wir haben, so lange auch der Niederschlag vor dem Filtrieren stehen mochte, nicht hin und wieder, sondern in allen unseren Fällen konstant Oxalsäuren im Filtrat gefunden, sogar in einem annähernd konstanten Verhältnis, und können daher das Verfahren von A u ten rie th und Bar th nicht empfehlen.

Prof. Salkowski machte mich nun noch darauf aufmerksam, daß ein altes Verfahren von Schultzen existiere, welches gleichfalls auf der Fällung des oxalsauren Kalks durch Ammoniak und Chlorcalcium beruht, jedoch den durch die Löslichkeit des oxalsauren Kalks verursachten Fehler dadurch vermeidet, daß der Harn mit dem Niederschlag möglichst zur Trockene gedampft und dann mit Alkohol und Äther behandelt wird, und forderte mich auf, dieses Verfahren mit der Extraktion der Oxalsäure aus saurer Lösung durch Äther zu kombinieren. Aus äußeren Gründen konnte ich diese Versuche nicht zum Abschluß bringen, jedoch wurde wenigstens eine Tatsache festgestellt.

$500 \mathrm{ccm}$ wurden mit Ammoniak und Chlorcalcium gefällt, dann, ohne zu filtrieren, stark eingedampft und mit Alkohol extrahiert, filtriert, der Niederschlag größtenteils auf das Filter gebracht, mit Alkohol nachgewaschen, dann mit Äther. In den eingedampften alkoholisch-ätherischen Auszügen ließ sich, wie zu vermuten war, keine Oxalsäure nachweisen. Der in der Schale und auf dem Filter befindliche Niederschlag wird dann in verdünnter Salzsäure gelöst und die Lösung mit Äther extrahiert usw.

Dieses Verfahren ist allerdings umständlicher als das ursprünglich von E. Salkowski angegebene, hat aber den Vorzug, daß die salzsaure Lösung offenbar reiner ist, weniger gefärbt und besser filtriert. $O b$ diese Vorzüge den Nachteil größerer Umständlichkeit aufwiegen, muß vorläufig dahingestellt bleiben. 